\title{
Experimental investigation of the tilt angle of turbulent structures in the core of fusion plasmas
}

\author{
J R Pinzón ${ }^{1,2}$, T Happel $^{1}$, P Hennequin ${ }^{3}$, C Angioni ${ }^{1}$, \\ T Estrada ${ }^{4}$, A Lebschy ${ }^{1}$, U Stroth ${ }^{1,2}$, the ASDEX Upgrade \\ Team $^{1}$ \\ ${ }^{1}$ Max-Planck-Institut für Plasmaphysik, Garching, Germany \\ ${ }^{2}$ Physik-Department E28, Technishe Universität München, Garching, Germany \\ ${ }^{3}$ Laboratoire de Physique de Plasmas, Ecole Polytechnique, Palaiseau, France \\ ${ }^{4}$ Laboratorio Nacional de Fusión, CIEMAT, Madrid, Spain \\ E-mail: javier.pinzon@ipp.mpg.de \\ January 2019
}

\begin{abstract}
.
The tilt angle of turbulent structures stands for the anisotropy of turbulence which is essential for understanding the dynamics of magnetized plasmas. It is a quantity predicted by theory and simulations, that provides information on the interplay between turbulence, micro-instabilities and plasma flows. A new method for measuring the tilt angle of turbulent structures in the core region of fusion plasmas using Doppler reflectometry is presented. First measurements of this type on the ASDEX Upgrade tokamak have shown a significant difference of tilt angle for different plasma conditions. The dominance of sheared $E \times B$ flows in determining the structure tilt is experimentally demonstrated for different turbulence regimes.
\end{abstract}

The dynamics of physical systems is frequently determined by turbulence. The macroscopic behaviour of fluids and plasmas can be closely related to the microscopic structures of turbulent fluctuations in density, temperature, and for plasmas, electric potential. In most plasmas, transport of particles, momentum and energy is governed by turbulence. Examples are found in astrophysics where turbulence is fundamental in the explosion mechanism of core-collapse supernovae [1], and also in magnetic fusion research where turbulence determines to a large extent the energy confinement time of the plasma [2].

In a magnetized plasma, turbulence can be considered as two-dimensional due to the high mobility of electrons and ions along the magnetic field. Therefore, in a simple description, turbulent structures can be described by an elliptical shape with characteristic size, elongation and tilt angle. This work addresses in particular the tilt angle of turbulent structures in the core of a fusion plasma. This quantity specifies the anisotropy of turbulence which is essential for the interaction with plasmas flows and instabilities [3]. 
Turbulence and $E \times B$ flows constitute a strongly coupled system [4]. A sheared $E \times B$ flow can tilt and stretch turbulent structures. If the $E \times B$ shear is strong enough, the structures are decorrelated leading to a reduction of the turbulence level [5]. At the same time, turbulence itself can generate $E \times B$ flows through the Reynolds stress mechanism, which requires a systematic variation of the structure tilt. This is the mechanism behind zonal flow generation [6], and residual stress contributions to transport of toroidal momentum [7].

Instabilities driving turbulence are characterized by a global mode structure whose symmetry is broken by the radial variation of plasma parameters [8]. The radial direction is defined across the magnetic surfaces. A radial variation of the phase velocity of the unstable modes induces a systematic tilt of the structures as shown by analytical results from the linear ballooning theory [9] and simulations [8, 9, 10]. Moreover, different mode structures have been observed in gyrokinetic simulations of ion-temperature-gradient (ITG) and trapped-electron (TEM) modes [8]. They are the dominant micro-instabilities in the core of fusion plasmas at the ion scale i.e. with perpendicular wavenumbers $k_{\perp}$ in the range $k_{\perp} \rho_{i} \approx 0.2-1\left(\rho_{i}=\sqrt{m_{i} T_{i}} /(e B)\right.$ is the ion Larmor radius). The perpendicular direction is defined tangential to magnetic surfaces and perpendicular to the magnetic field.

Despite the importance of the tilt angle, its direct measurement in fusion plasmas remains challenging. Measurements have been provided using gas puff imaging [11, 12], Langmuir probes $[13,14]$, and beam emission spectroscopy [15, 16]. This letter presents a new method to measure the tilt angle of turbulent structures using Doppler reflectometry, which is a standard diagnostic technique based on the scattering of microwaves at electron density fluctuations [17]. It has the advantage of being noninvasive and applicable in the core of fusion plasmas where the best suited modelling tools, e.g. gyrokinetic simulations [18], are available. The new method is applied on discharges of the ASDEX Upgrade (AUG) tokamak for two parameter sets, and tilt angle measurements in the core region are reported for the first time. From the comparison of experimental measurements with simple models, the impact of mode structure and $E \times B$ shear on the tilt angle is assessed.

A low confinement plasma in lower single null magnetic configuration has been investigated. The plasma current is $0.8 \mathrm{MA}$ and the toroidal magnetic field on-axis is $2.5 \mathrm{~T}$. A neutral beam injection heating (NBI) power of $0.8 \mathrm{MW}$ is applied during the complete plasma discharge. After an initial phase with only NBI external heating, 1.5 MW electron cyclotron resonance heating $(\mathrm{ECRH})$ power is added. In the following, the two phases are labelled as "NBI phase" and "ECRH phase", respectively. The different heating systems are used to vary the electron to ion heat flux ratio and are intended to induce different turbulence regimes. In addition, short NBI blips are applied for obtaining charge exchange recombination spectroscopy (CXRS) measurements [19].

Figure 1 shows radial profiles of the main plasma parameters. The radial coordinate $\rho_{\text {pol }}$ is the normalized poloidal flux radius. The plasma density in (a) is obtained from Thomson scattering [20] and lithium beam diagnostics [21]. The electron temperature 


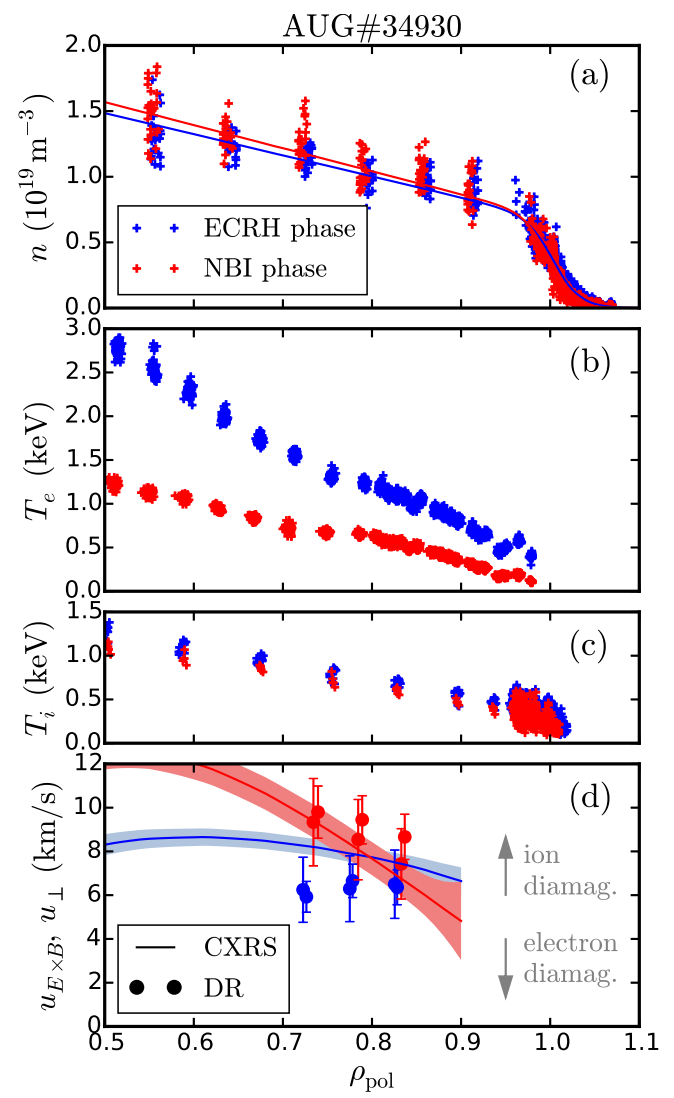

Figure 1. Radial profiles for ECRH and NBI phases of (a) plasma density, (b) electron temperature, (c) ion temperature and (d) plasma velocity. The $E \times B$ velocity is obtained from CXRS measurements (solid line) and the turbulence velocity $u_{\perp}$ from Doppler reflectometry measurements (circles).

profiles in (b) are obtained from Thomson scattering and electron cyclotron emission measurements [22]. The additional electron heating in the ECRH phase results in a higher electron temperature. The ion temperature in (c) obtained from CXRS measurements is rather similar for both phases. Nevertheless, the electron-ion collisional heat transfer increases slightly the ion temperature in the ECRH phase. The $E \times B$ drift velocity $\left(u_{E \times B}\right)$ computed from CXRS measurements [19] is shown in Fig. 1d with solid lines. In the ECRH phase the velocity is approximately constant at about $8 \mathrm{~km} / \mathrm{s}$, whereas the NBI phase shows a strong shear as the velocity increases from about $5 \mathrm{~km} / \mathrm{s}$ at $\rho_{\text {pol }}=0.9$ to $10 \mathrm{~km} / \mathrm{s}$ at $\rho_{\text {pol }}=0.7$.

The experimental technique used in this letter for investigating turbulence is Doppler reflectometry (DR) [17]. A microwave beam is obliquely sent into the plasma from an antenna located outside. The beam propagates in the plasma and is reflected at the cutoff layer, where the refractive index is minimum and backscattering at density fluctuations with $k_{\perp}$ fulfilling the Bragg condition is strong. The backscattered wave propagates back to the antenna where it is detected. The frequency of the backscattered wave is Doppler shifted due to the perpendicular propagation velocity of the turbulent structures $u_{\perp}=u_{E \times B}+v_{\mathrm{ph}}$. It corresponds to the $E \times B$ flow and an intrinsic phase 

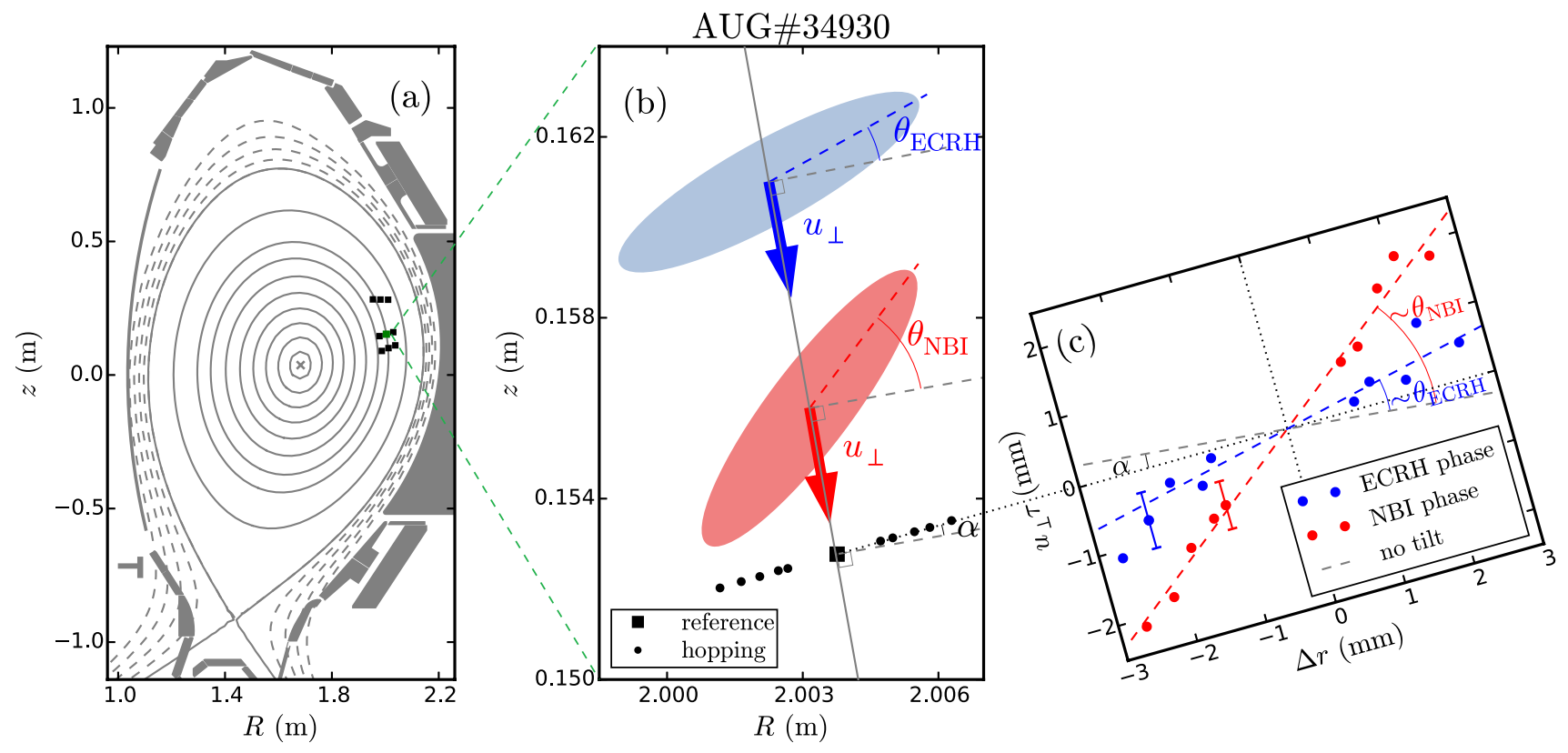

Figure 2. (a) Doppler reflectometry measurement positions on the AUG cross-section. (b) Enlargement showing the reference and hopping channel measurement positions in detail. Turbulent structures are schematically depicted, along with their tilt angle and propagation direction. (c) Time delay $\tau$ multiplied by $u_{\perp}$ as a function of the radial separation $\Delta r$. The grey dashed line indicates $u_{\perp} \tau$ for radially aligned structures, hence the angle formed by the measurement and this line gives approximately the tilt angle $\theta$.

velocity $v_{\mathrm{ph}}$ in the plasma frame, which is characteristic of the type of turbulence. The frequency Doppler shift $f_{D}$ provides a measurement of $u_{\perp}=2 \pi f_{D} / k_{\perp}$.

Radial correlation Doppler reflectometry [23, 24] uses two beams probing simultaneously at radially displaced positions. The probing frequency of one beam (referred to as reference channel) is fixed whereas the frequency of the other one (hopping channel) is scanned. In the standard technique, the correlation level of the reflectometer signals and its dependence on the radial separation $\Delta r$ are used for estimating the turbulent radial correlation length $[23,24]$. In this letter, the time delay $\tau$ obtained from the correlation of the two reflectometer signals is used for measuring the tilt angle of turbulent structures.

Doppler reflectometry measurements have been performed with two V-band channels [25] coupled in X-mode polarization to the antenna system installed in AUG [26]. For the reference channel, probing frequencies of $69.0,70.5$ and $72.0 \mathrm{GHz}$ (during $50 \mathrm{~ms}$ each) are used for three angles of incidence of the probing beam. The scattering position and the probed perpendicular wavenumber $k_{\perp}$ are obtained with the ray tracing code TORBEAM [27] and using fits to the density profiles (solid lines in Fig. 1a). The scattering positions are depicted with black squares on the AUG crosssection in Fig. 2a. Closed and open magnetic surfaces are depicted with solid and dashed grey lines, respectively. Measurements are in the radial range $\rho_{\text {pol }}=0.70-0.84$ and at perpendicular wavenumbers $k_{\perp}$ within $3-8 \mathrm{~cm}^{-1}$. 
Results of the turbulence propagation velocity are shown with circles in Fig. 1d. Although Doppler reflectometry measurements show a similar behaviour as CXRS data, there are systematic differences that suggest finite phase velocities $v_{\text {ph }}$ with respect to $u_{E \times B}$. In the NBI phase, a positive $v_{\mathrm{ph}}$ in the ion diamagnetic direction indicates iondriven turbulence, i.e. ITG. Contrarily in the ECRH phase, a negative $v_{\text {ph }}$ in the electron diamagnetic direction indicates electron-driven turbulence, i.e. TEM. The dispersion of data may be a signature of a small variation of $v_{\mathrm{ph}}$ with $k_{\perp}$.

For the correlation measurements, the hopping channel probes 10 frequencies $(5 \mathrm{~ms}$ each) in a $0.15 \mathrm{GHz}$ range around the reference channel frequency. In the following, the method to measure the tilt angle is presented for the case zoomed in Fig. 2b. The measurement position of the reference and hopping channels are depicted with a square and circles, respectively. Note that due to the oblique incidence of the probing beam, the measurement positions are not aligned along the radial direction (dashed grey line), but form an angle $\alpha$ with respect to it. The time delays $\tau$ have been obtained from the time lag at the maximum of the cross-correlation function of reference and hopping channel signals. $\tau$ values have been multiplied by $u_{\perp}$ and plotted as function of the radial separation in Fig. 2c. A linear dependence of $u_{\perp} \tau$ on $\Delta r$ is observed. The dashed lines are linear fits to the data.

The measured time delays indicate that turbulent structures are tilted as depicted schematically in Fig. 2b. As the structures propagate downwards, they are "seen" first by the hopping channel for $\Delta r<0$ and later by the reference one, obtaining a negative time delay. Complementary, for $\Delta r>0$ the time delay is positive. The larger slope obtained in the NBI phase implies a stronger structure tilt than in the ECRH phase. In contrast, if structures are aligned with the measurement positions, a zero time delay is obtained as structures are simultaneously seen by reference and hopping channels. Figure 2c has been rotated accordingly.

Moreover, if structures are radially aligned, the grey dashed line is to be obtained in Fig. 2c because of the misalignment of the measurement positions by an angle $\alpha$. Consequently, the tilt angle of the turbulent structures $\theta$, defined with respect to the radial direction, can be approximated by the angle formed by the measurements and the grey dashed line as indicated in Fig. 2c. This representation is intuitive and shows the principle of the measurement method. A more careful analysis of the geometry allows to calculate accurately the tilt angle from

$$
\frac{u_{\perp} \tau}{\Delta r}=\tan \theta-\tan \alpha .
$$

Note that $u_{\perp} \tau / \Delta r$ corresponds to the slope of the linear fits in Fig. 2c.

The values of $u_{\perp} \tau / \Delta r$ obtained from all reference positions are shown as a function of the angle $\alpha$ in Fig. 3. For each phase, data are satisfactorily fitted with Eq. 1 and with a constant tilt angle (solid line). The tilt angles obtained from the fit are

$$
\theta_{\mathrm{ECRH}}=(18 \pm 7)^{\circ}, \quad \theta_{\mathrm{NBI}}=(44 \pm 6)^{\circ} .
$$

Schematic structures with the corresponding tilt have been depicted in Fig. 2b. 


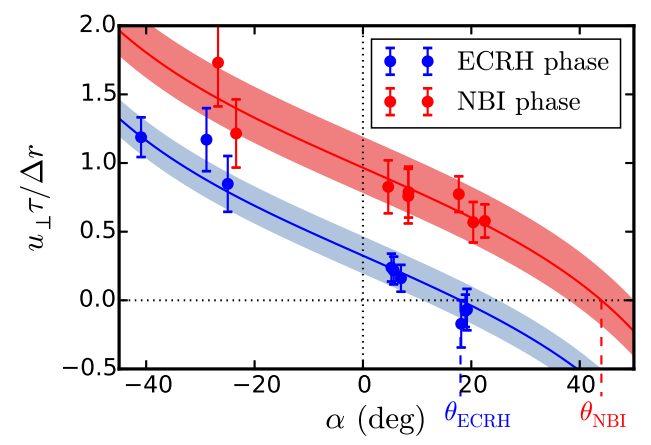

Figure 3. $u_{\perp} \tau / \Delta r$ values obtained from all reference positions as a function of the angle $\alpha$. The solid lines depict the fit with Eq. 1 from which the average tilt angle is obtained.
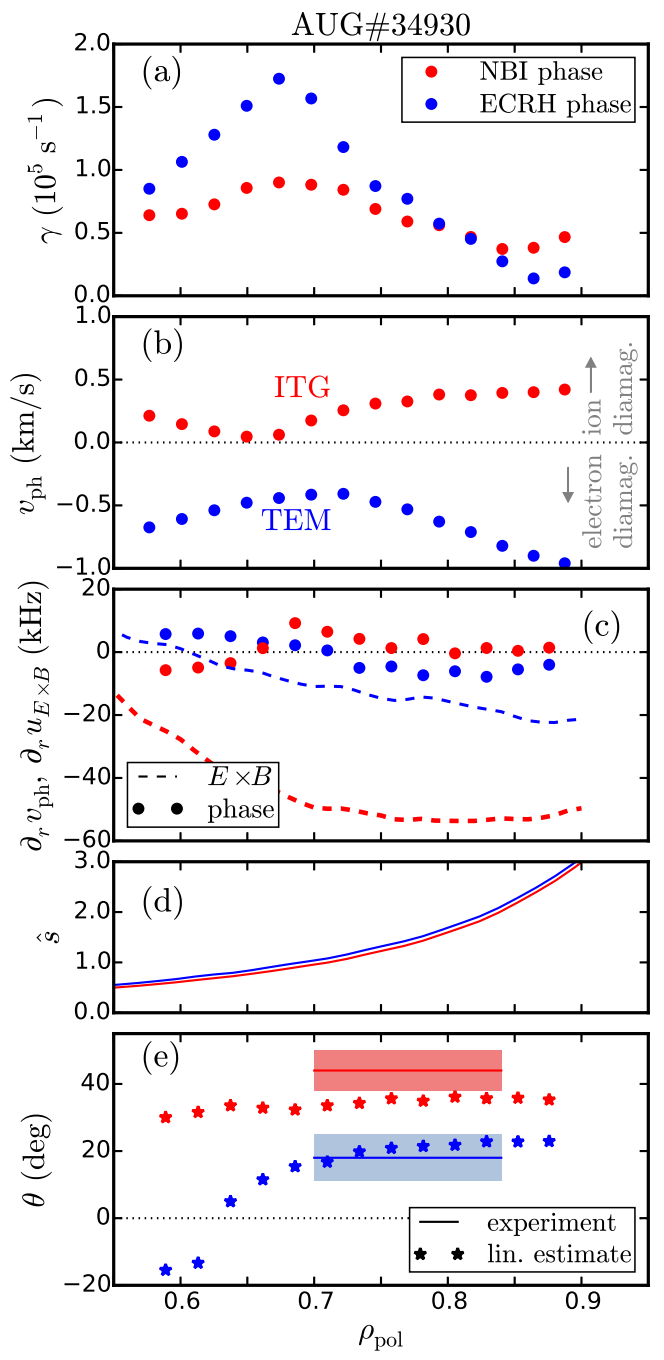

Figure 4. (a) Linear growth rate and (b) phase velocity obtained with GKW simulations. (c) $E \times B$ (dashed lines) and phase velocity (circles) shear. (d) Magnetic shear $\hat{s}$. (e) Tilt angle estimated with Eq. 3 (stars) and experimental measurements (solid line). 
The significant difference between the tilt angle obtained for both phases proves that the method is applicable and that it provides results sensitive to plasma parameters. Further details on the method, its applicability to general experimental conditions, diagnostic effects related to the structure life time and finite scattering volume, will be presented in a future publication [28]. In the rest of this letter, the experimental measurements are compared with results from linear ballooning theory [10] in order to obtain a first estimate of the physical mechanisms which could potentially explain the observed differences in tilt angle. In this framework, the tilt angle is estimated by [10]

$$
\theta \approx-\operatorname{sign}\left[\hat{s}\left(\partial_{r} v_{\mathrm{ph}}+\partial_{r} u_{E \times B}\right)\right]\left|\frac{\partial_{r} v_{\mathrm{ph}}+\partial_{r} u_{E \times B}}{2 \gamma \hat{s}}\right|^{1 / 3},
$$

where $\partial_{r}$ denotes the radial derivative, $\gamma$ the growth rate of the instability and $\hat{s}$ the magnetic shear. The effect of the instability mode structure is included through the shear of the mode velocity $\partial_{r} v_{\mathrm{ph}}$, and the effect of the $E \times B$ shear through $\partial_{r} u_{E \times B}$. The addition of both terms in the numerator indicates the competition of both physical mechanisms in the determination of the tilt angle.

In order to obtain the linear growth rate and phase velocities, the two plasma phases have been investigated with linear local gyrokinetic simulations using the code GKW [29]. The experimental density and temperature profiles have been used as an input for the code. A linear stability analysis has been performed in the radial range of interest for the perpendicular wavenumber $k_{\perp} \approx 3.5 \mathrm{~cm}^{-1}$ consistent with the experimental measurements and corresponding to the ion scale $\left(k_{\perp} \rho_{i} \approx 0.7\right)$. The growth rates and phase velocities are presented in Figs. $4 a$ and b. The phase velocity points in the ion and electron diamagnetic directions for the NBI and ECRH phases, respectively. This confirms ITG and TEM dominated turbulence regimes, in agreement with the experimental indication of phase velocity in Fig. 1d.

The velocity shear in Fig. $4 \mathrm{c}$ is obtained from GKW data $\left(\partial_{r} v_{\mathrm{ph}}\right)$ and from CXRS measurements $\left(\partial_{r} u_{E \times B}\right)$. The $E \times B$ shear is larger than the phase velocity shear in both cases, indicating that $E \times B$ shear is the dominant effect. In the NBI phase, a factor of 10 suggests that the tilt angle is determined by $E \times B$ shear only. In the ECRH phase, although the $E \times B$ shear dominates, the mode structure could still contribute weakly to the tilt angle.

The magnetic shear $\hat{s}$ plays also a role in determining the tilt angle as evidenced by Eq. 3. Magnetic shear profiles obtained from equilibrium reconstruction are shown in Fig. $4 \mathrm{~d}$ for both phases. Since there is no significant difference, magnetic shear cannot account for the tilt angle change between both phases.

For the estimation of the tilt angle from Eq. 3, the maximum $\gamma$ value in the radial scan corresponding to the most unstable mode has been taken for each phase. The results are shown in Fig. 4e (stars), together with the experimental measurements (cf. Eq. 2). The linear estimate recovers qualitatively the stronger tilt for the NBI phase, moreover the values for the ECRH phase are within the experimental errorbars. Since Eq. 3 gives just an estimate for the linear case, an exact quantitative agreement is not 
expected. Nevertheless, the similarity of the magnitudes indicate that for the plasmas under investigation, the tilt angle is predominantly induced by the $E \times B$ shear.

For future studies, a better assessment of the mode structures can be achieved with global simulations $[9,10,8]$. Moreover the competition of both effects, mode structure and $E \times B$ shear, as well as non-linear interactions can be self-consistently treated with more advanced tools as non-linear global gyrokinetic simulations. It is possible that for scenarios with low $E \times B$ shear, the tilt angle measurement might provide a signature of the dominant turbulence regime.

In this letter, a novel measurement method of the tilt angle of turbulent structures has been proposed, and measurements in the core of the ASDEX Upgrade Tokamak have been presented for the first time. The comparison of the experimental measurements with simple models has demonstrated the dominant effect of sheared $E \times B$ flows on the structure tilt for ITG and TEM dominated turbulence. In the future, the systematic application of this technique can provide radially and temporally resolved measurements, with a few $\mathrm{mm}$ resolution and in the $\mathrm{kHz}$ range. This will enable a wealth of turbulence studies, for example of the local Reynolds stress and zonal flows, both fundamental elements for understanding the interactions of turbulence in plasmas.

\section{Acknowledgments}

This work has been carried out within the framework of the EUROfusion Consortium

and has received funding from the Euratom research and training program 2014-2018 and 2019-2020 under grant agreement No 633053. The views and opinions expressed herein do not necessarily reflect those of the European Commision.

\section{References}

[1] D Radice, E Abdikamalov, C D Ott, P Mösta, S M Couch, and L F Roberts. Turbulence in core-collapse supernovae. Journal of Physics G: Nuclear and Particle Physics, 45(5):053003, 2018.

[2] E J Doyle, W A Houlberg, Y Kamada, V Mukhovatov, T H Osborne, A Polevoi, G Bateman, J W Connor, J G Cordey, T Fujita, X Garbet, T S Hahm, L D Horton, A E Hubbard, F Imbeaux, F Jenko, J E Kinsey, Y Kishimoto, J Li, T C Luce, Y Martin, M Ossipenko, V Parail, A Peeters, T L Rhodes, J E Rice, C M Roach, V Rozhansky, F Ryter, G Saibene, R Sartori, A C C Sips, J A Snipes, M Sugihara, E J Synakowski, H Takenaga, T Takizuka, K Thomsen, M R Wade, H R Wilson, ITPA Transport Physics Topical Group, ITPA Confinement Database and Modelling Topical Group, and ITPA Pedestal and Edge Topical Group. Chapter 2: Plasma confinement and transport. Nuclear Fusion, 47(6):S18, 2007.

[3] P W Terry. Suppression of turbulence and transport by sheared flow. Rev. Mod. Phys., 72:109-165, Jan 2000.

[4] U Stroth, P Manz, and M Ramisch. On the interaction of turbulence and flows in toroidal plasmas. Plasma Physics and Controlled Fusion, 53(2):024006, 2011.

[5] H Biglari, P H Diamond, and P W Terry. Influence of sheared poloidal rotation on edge turbulence. Physics of Fluids B: Plasma Physics, 2(1):1-4, 1990.

[6] P H Diamond, S-I Itoh, K Itoh, and T S Hahm. Zonal flows in plasma - a review. Plasma Physics and Controlled Fusion, 47(5):R35, 2005. 
[7] C Angioni, R M McDermott, F J Casson, E Fable, A Bottino, R Dux, R Fischer, Y Podoba, T Pütterich, F Ryter, and E Viezzer. Intrinsic toroidal rotation, density peaking, and turbulence regimes in the core of tokamak plasmas. Physical Review Letters, 107:215003, Nov 2011.

[8] Y Camenen, Y Idomura, S Jolliet, and A G Peeters. Consequences of profile shearing on toroidal momentum transport. Nuclear Fusion, 51(7):073039, 2011.

[9] J Y Kim, Y Kishimoto, M Wakatani, and T Tajima. Poloidal shear flow effect on toroidal ion temperature gradient mode: A theory and simulation. Physics of Plasmas, 3(10):3689-3695, 1996.

[10] Y Kishimoto, J-Y Kim, W Horton, T Tajima, M J LeBrun, and H Shirai. Toroidal mode structure in weak and reversed magnetic shear plasmas and its role in the internal transport barrier. Plasma Physics and Controlled Fusion, 41(3A):A663, 1999.

[11] I Shesterikov, Y Xu, G R Tynan, P H Diamond, S Jachmich, P Dumortier, M Vergote, M Van Schoor, G Van Oost, and Team TEXTOR. Experimental evidence for the intimate interaction among sheared flows, eddy structures, reynolds stress, and zonal flows across a transition to improved confinement. Physical Review Letters, 111:055006, Aug 2013.

[12] S J Zweben, D P Stotler, F Scotti, and J R Myra. Two-dimensional turbulence cross-correlation functions in the edge of NSTX. Physics of Plasmas, 24(10):102509, 2017.

[13] T A Carter and J E Maggs. Modifications of turbulence and turbulent transport associated with a bias-induced confinement transition in the Large Plasma Device. Physics of Plasmas, 16(1):012304, 2009.

[14] D Guszejnov, A Bencze, S Zoletnik, and A Krämer-Flecken. Determination of structure tilting in magnetized plasmastime delay estimation in two dimensions. Physics of Plasmas, 20(6):062303, 2013.

[15] M W Shafer, R J Fonck, G R McKee, C Holland, A E White, and D J Schlossberg. 2D properties of core turbulence on DIII-D and comparison to gyrokinetic simulations. Physics of Plasmas, 19(3):032504, 2012.

[16] M F J Fox, F van Wyk, A R Field, Y c Ghim, F I Parra, and A A Schekochihin and. Symmetry breaking in MAST plasma turbulence due to toroidal flow shear. Plasma Physics and Controlled Fusion, 59(3):034002, jan 2017.

[17] M Hirsch, E Holzhauer, J Baldzuhn, B Kurzan, and B Scott. Doppler reflectometry for the investigation of propagating density perturbations. Plasma Physics and Controlled Fusion, 43(12):1641, 2001.

[18] X Garbet, Y Idomura, L Villard, and T H Watanabe. Gyrokinetic simulations of turbulent transport. Nuclear Fusion, 50(4):043002, 2010.

[19] A Lebschy, R M McDermott, C Angioni, B Geiger, D Prisiazhniuk, M Cavedon, G D Conway, R Dux, M G Dunne, A Kappatou, T Pütterich, U Stroth, E Viezzer, and the ASDEX Upgrade Team. Measurement of the complete core plasma flow across the LOC-SOC transition at ASDEX Upgrade. Nuclear Fusion, 58(2):026013, 2018.

[20] H Murmann, S Götsch, H Röhr, H Salzmann, and K H Steuer. The Thomson scattering systems of the ASDEX Upgrade tokamak. Review of Scientific Instruments, 63(10):4941-4943, 1992.

[21] R Fischer, E Wolfrum, J Schweinzer, and the ASDEX Upgrade Team. Probabilistic lithium beam data analysis. Plasma Physics and Controlled Fusion, 50(8):085009, 2008.

[22] S K Rathgeber, L Barrera, T Eich, R Fischer, B Nold, W Suttrop, M Willensdorfer, E Wolfrum, and the ASDEX Upgrade Team. Estimation of edge electron temperature profiles via forward modelling of the electron cyclotron radiation transport at ASDEX Upgrade. Plasma Physics and Controlled Fusion, 55(2):025004, 2013.

[23] J Schirmer, G D Conway, E Holzhauer, W Suttrop, H Zohm, and the ASDEX Upgrade Team. Radial correlation length measurements on ASDEX Upgrade using correlation Doppler reflectometry. Plasma Physics and Controlled Fusion, 49(7):1019, 2007.

[24] F Fernández-Marina, T Estrada, and E Blanco. Turbulence radial correlation length measurements using Doppler reflectometry in TJ-II. Nuclear Fusion, 54(7):072001, 2014. 
[25] R Sabot, P Hennequin, and L Colas. Fluctuation measurements and their link with transport on tore supra. Fusion Science and Technology, 56(3):1253-1272, 2009.

[26] T Happel, A Bañón Navarro, G D Conway, C Angioni, M Bernert, M Dunne, E Fable, B Geiger, T Görler, F Jenko, R M McDermott, F Ryter, U Stroth, and the ASDEX Upgrade Team. Core turbulence behavior moving from ion-temperature-gradient regime towards trapped-electronmode regime in the ASDEX Upgrade tokamak and comparison with gyrokinetic simulation. Physics of Plasmas, 22(3):032503, 2015.

[27] E Poli, A Bock, M Lochbrunner, O Maj, M Reich, A Snicker, A Stegmeir, F Volpe, N Bertelli, R Bilato, G D Conway, D Farina, F Felici, L Figini, R Fischer, C Galperti, T Happel, Y R Lin-Liu, N B Marushchenko, U Mszanowski, F M Poli, J Stober, E Westerhof, R Zille, A G Peeters, and G V Pereverzev. Torbeam 2.0, a paraxial beam tracing code for electron-cyclotron beams in fusion plasmas for extended physics applications. Computer Physics Communications, 225:36- 46, 2018.

[28] J R Pinzón, T Estrada, T Happel, P Hennequin, E Blanco, U Stroth, the ASDEX Upgrade team, and the TJ-II team. Measurement of the tilt angle of turbulent structures in magnetically confined plasmas using Doppler reflectometry. To be submitted to Plasma Physics and Controlled Fusion, 2019.

[29] A G Peeters, Y Camenen, F J Casson, W A Hornsby, A P Snodin, D Strintzi, and G Szepesi. The nonlinear gyro-kinetic flux tube code GKW. Computer Physics Communications, 180(12):2650$2672,2009$. 